\title{
étude expérimentale de la bifurcation dans les roches
}

\author{
experimental study of bifurcation in rocks
}

\author{
F.J. SANTARELLI \\ Elf Aquitaine*
}

Rev. Franç. Géotech. n 50, pp. $61-70$ (janvier 1990)

\section{Résumé}

Cet article présente divers aspects liés à l'étude expérimentale de la bifurcation dans les roches. Dans un premier temps, il présente un catalogue des différentes techniques expérimentales pour détecter la bifurcation puis il les compare. Dans un second temps, une étude de 29 tests triaxiaux sur un grès est présentée. Les applications industrielles de ces méthodes sont soulignées.

\footnotetext{
Abstract

This paper presents various aspects of the experimental study of bifurcation in rocks. Firstly, it presents a review of the various experimental techniques used to detect bifurcation and compares the results they give. Second, a series of 29 triaxial tests on a sandstone is presented as an application. Industrial applications are emphasized.
} 


\section{INTRODUCTION}

Initialement développées pour les sols, les analyses en bifurcations sont appliquées depuis quelques années aux géomatériaux cohérents et notamment aux roches (VARDOULAKIS, 1984 ; VARDOULAKIS, SULEM et GUENOT, 1988). Devant le caractère prometteur de l'application de cette technique à la stabilité d'ouvrages souterrains (MAURY, 1987), il est intéressant d'étudier expérimentalement le processus de rupture dans l'optique de la théorie des bifurcations. On notera que les termes seuil de bifurcation et point de bifurcation seront utilisés dans cette étude pour désigner le point critique sur les courbes effortsdéformations où le concept de bifurcation développé par les auteurs mentionnés au-dessus est susceptible de s'appliquer. Ce vocable est utilisé seulement par référence à ces théories sans préjuger de l'applicabilité de celles-ci.

Les buts de cet article sont :

- de définir où l'on peut fixer le niveau de contraintes correspondant au point de bifurcation au cours du processus de rupture pendant l'essai triaxial de révolution ;

- de cataloguer les différentes méthodes expérimentales pour détecter ce point de bifurcation et de les comparer ;

- d'illustrer par un exemple ce que peut apporter une telle étude expérimentale ;

- de présenter des observations expérimentales qui puissent permettre d'apporter des éléments de réponse aux questions qui se posent lorsque l'on tente d'appliquer les analyses en bifurcations aux matériaux consolidés.

On insistera sur l'aspect industriel, tant au niveau interprétation des résultats que mise en œuure des techniques de laboratoire.

\section{LES DIFFÉRENTES TECHNIQUES D'ÉTUDES}

\subsection{Le modèle de PATERSON}

Dans son ouvrage, PATERSON (1978) indique quatre étapes successives lors de la rupture d'une éprouvette cylindrique de roche par plan de cisaillement lors de l'essai triaxial de révolution (fig. 1).

- Tout d'abord une fermeture élastique ou inélastique des pores du matériau. Cette fermeture qui se traduit par la courbure inverse de la courbe contraintedéformation tend à disparaître lorsqu'on applique une pression de confinement à l'éprouvette.

- Une phase II où la plus grande partie des déformations est élastique et où la courbe effort-déformation est linéaire. Il serait incorrect de conclure de cette linéarité et de cette élasticité que la roche est un matériau linéaire élastique. En effet, de nombreux auteurs ont montré que la pente de la courbe effort-défor-

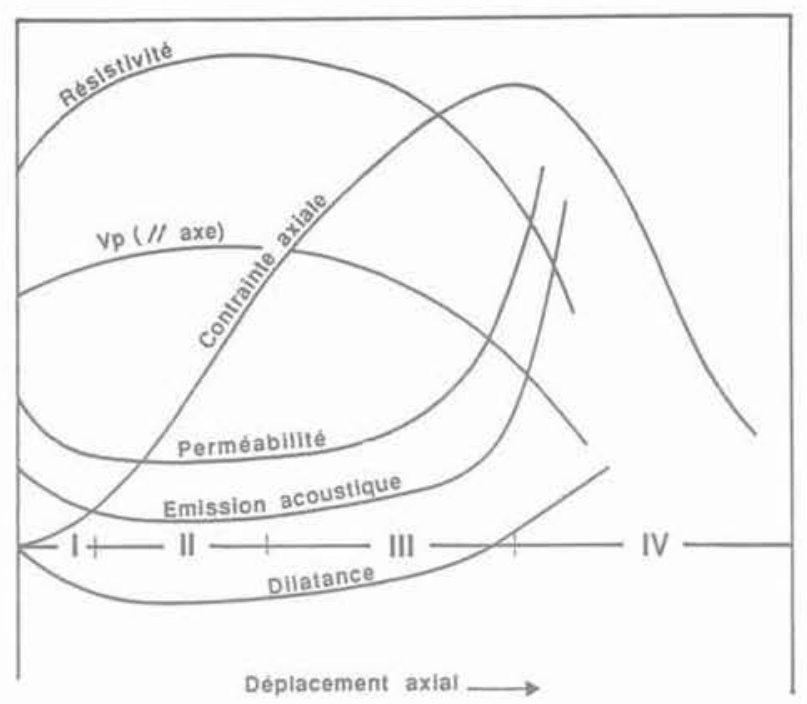

Fig. 1. - Les 4 stades de la déformation d'une éprouvette au cours d'un essai triaxial de révolution. D'après PATERSON (1978)

Fig. 1. - The four deformation stages of a rock specimen tested in triaxial conditions. After Paterson (1978)

mation ou module d'Young s'accroîssait lorsque l'on accroît la pression de confinement (KULHAWY, 1975)

- Au-delà d'un certain seuil dit seuil de microfissuration (phase III), des microfissurations commencent à se développer dans la direction parallèle à l'axe qui est la direction de la contrainte principale majeure. On insistera sur le fait que cette microfissuration est uniformément répartie dans l'échantillon à l'exception des zones près des plateaux, influencées par le frettage. De nombreuses méthodes ont été utilisées pour étudier ce développement de microfissures. Outre l'observation directe, ce sont la mesure directe ou indirecte de la déformation volumétrique de l'échantillon, l'enregistrement de l'émission acoustique, la mesure des célérités des ondes et de leurs atténuations voire la mesure de la perméabilité à l'air ou la résistivité électrique. Toutes ces mesures indiquent que le seuil de microfissuration se situe souvent entre 30 et $60 \%$ du pic et que la microfissuration se poursuit bien au-delà du pic.

- Au-delà d'un autre seuil que PATERSON (O.C.) appelle le seuil de localisation (phase IV), les déformations dans l'éprouvette deviennent inhomogènes et se localisent dans une bande mince. Les premières microfissures inclinées sur l'axe se développent alors et se concentrent dans une zone proche du plan de rupture (RAMEZ, 1967). Ce seuil de localisation est le véritable point d'initiation de la rupture car toute la partie post-pic correspond à l'endommagement progressif de la zone de déformation localisée donnant naissance au traditionnel plan de cisaillement. PATERSON (1978) insiste sur le fait que dans la partie postlocalisation de la courbe charge-déplacement, il n'est pas possible de calculer les contraintes et les déformations directement dans la mesure où les déformations ne sont plus homogènes. Sur le sujet, on pourra aussi se reporter à l'état des connaissances fait par READ et HEGEMEIER (1984). PATERSON place ce 
seuil de localisation au pic mais reconnaît que des études complémentaires sont nécessaires pour conclure.

\subsection{Les méthodes de détection du seuil de localisation}

Expérimentalement, la détection du seuil de localisation devra se concentrer sur la mise en évidence de l'un des deux phénomènes qui se produit au seuil de localisation. Ces deux phénomènes sont :

- l'apparition de déformations inhomogènes ;

- une concentration des microfissures dans une bande de l'éprouvette et apparition des premières microfractures inclinées sur la verticale.

Si l'on garde à l'esprit ces deux points précis et si l'on se livre à une étude de la bibliographie de mécanique des roches expérimentale, on se rend compte que la détermination du seuil de localisation est pratiquée depuis longtemps. Pour ce faire, de nombreuses méthodes expérimentales ont été utilisées qui peuvent se regrouper autour des deux phénomènes clés mentionnés ci-dessus : les microfracturations et les déformations. Ces deux groupes de techniques seront présentées, tour à tour, ci-dessous.

Parmi les méthodes qui consistent à étudier les microfissures, la plus directe consiste à charger des éprouvettes jusqu'à un certain point de la courbe chargement-déplacement puis à la décharger et à confectionner un certain nombre de sections polies et de lames minces qui seront étudiées sous le microscope soit en réflexion (BRADY, 1974) soit en transmission (FRIEDMAN, PERKINS et GREEN, 1970), soit encore, sous le microscope électronique à balayage (KRANZ, 1979 et WONG, 1982). Ces techniques permettent d'étudier la morphologie des microfissures, leur orientation et leur localisation. Cette technique offre l'avantage de pouvoir être utilisée sur pratiquement n'importe quel essai, que ce soit uniaxial, triaxial de révolution, biaxial au triaxial vrai. Pourtant elle a le désavantage majeur de nécessiter des tests relativement nombreux pour déterminer précisément le seuil de localisation. Quoique l'usage de répliques de la surface de l'échantillon tourne cette difficulté (TOU. RENQ, 1967). L'utilisation d'une telle méthode dans un contexte industriel semble néanmoins difficile.

Une autre méthode consiste à enregistrer les événements acoustiques de la roche qui se rompt et à les localiser (SCHOLZ, 1968; SONDERGELD et ESTEY, 1981 et YANAGIDANI et al, 1985). Cette technique peut être utilisée pour tous les types d'essai à condition que le bruit de fond provoqué par le fonctionnement de la presse puisse être filtré. La méthode nécessite un équipement spécifique, (acquisition et interprétation de données). Elle est courante dans le contrôle non destructif des matériaux composites.

Enfin, une dernière méthode consiste à mesurer la vitesse des ondes $\mathrm{P}$ parallèle à l'axe de chargement (FOURMAINTRAUX, 1970 ; THILL, 1972 ; RAO et RAMANA, 1974 et LOCKNER, WALSH et BYERLEE, 1977). Cette vitesse des ondes reste pratiquement inchangée lorsque les microfissures qui se déve- loppent dans l'échantillon restent parallèles à la direction de propagation de l'onde. Par contre, cette vitesse commence à chuter dès que des microfissures inclinées sur l'axe se développent. Cette méthode est adaptable aux divers essais et surtout relativement facile à mettre en cuvre.

L'autre façon d'aborder la détection du seuil de localisation est de mesurer précisément la déformation de l'échantillon. Outre la stéréophotogrammétrie utilisée avec succès pour les sols par DESRUES (1987) et par TORRENTI (1987) pour les bétons, on peut citer trois méthodes :

- la première consiste à mesurer la diffraction d'une onde lumineuse dans une fente formée d'une part par un côté de l'échantillon que l'on teste et d'autre part, par une plaque d'acier (LIU et LIVANOS, 1976). La figure de diffraction dépendant de la largeur de la fente, il est alors possible de remonter à la déformation de l'échantillon. Cette méthode qui n'est guère utilisable que pour l'essai uniaxial, est citée ici pour mémoire :

- la méthode la plus pratiquée en mécanique des roches est sans aucun doute l'interférométrie holographique. SPETZLER et MARTIN (1974) et SPETZLER, SCHOLZ et LU (1974) l'utilisent lors d'essais uniaxiaux, SOGA et al (1978) lors d'essais triaxiaux, et SOBOLEV, SPETZLER et SALOV (1978) lors d'essais biaxiaux. Cette méthode qui consiste à superposer une photographie holographique de l'échantillon sous charge et de la comparer avec une photographie de l'échantillon non chargé permet de tracer une véritable carte des déformations d'une surface plane de l'éprouvette. Cette technique qui demande un certain équipement peut se révéler assez onéreuse à mettre en place sur une cellule triaxiale classique (saphir optique, choix du fluide de confinement, changement de la géométrie des éprouvettes...). Elle semble donc, elle aussi, assez difficile à mettre en œuvre dans un laboratoire industriel;

- une dernière méthode consiste simplement à "multiplier» le nombre de capteurs mesurant les déformations de l'échantillon. Ces capteurs peuvent être des extensomètres (HADLEY, 1975 et YANAGIDANI et al, 1985) voire même des capteurs de déplacement tel le * cantilever * utilisé par SANTA. RELLI (1987). Il convient ensuite d'interpréter ces mesures et de faire apparaître les effets d'un champ de déplacement inhomogène. L'avantage de cette technique est qu'elle utilise du matériel très couramment utilisé dans les laboratoires industriels.

\subsection{Comparaison des résultats de ces méthodes}

Il n'existe pas d'étude spécifique systématique visant à comparer les diverses techniques évoquées dans la section précédente et l'on doit se contenter d'une approche très générale. Le tableau 1 résume les résultats de toutes les études citées dans la section précédente. Elle révèle que toutes ont placé le seuil de localisation avant le pic et ce pour des valeurs du rapport « Loc » de la contrainte axiale au pic des contraintes variant entre 60 et $99 \%$. Une telle disper- 
sion peut naturellement être expliquée par la grande variété des roches testées et par celle des essais considérés.

Cependant, on constate que pour une même roche (granite de Westerley) et un même essai (triaxial), la dispersion des valeurs de « Loc » est aussi importante (de 60 à $99 \%$ ). Cette constatation indique que lune de causes majeures de la dispersion des valeurs de "Loc » est la sensibilité de la technique utilisée pour la détermination du seuil de localisation. Au vu de cette remarque, on aurait tendance à conclure que seules les faibles valeurs de \& Loc » doivent être conservées dans le tableau 1.

Pourtant, il convient de garder à l'esprit que SPETZLER, SCHOLZ et LU (1974) ont montré comment des problèmes de frettage et d'application de la charge mal maîtrisés conduisaient à des localisations très précoces qui pouvaient être retardées lorsque les causes expérimentales qui les engendraient étaient corrigées.

Au vu de cette remarque, il convient de considérer avec prudence les résultats indiquant des seuils de localisation très bas, dans la mesure où ils semblent plus à même de représenter des problèmes avec la machine d'essai que des propriétés réelles de la roche.

\subsection{Conclusion}

On retiendra de l'étude précédente les points suivants :

- le seuil de localisation est toujours situé avant le pic et la contrainte correspondante est entre $60 \%$ et $99 \%$ de celle du pic ;

- au seuil de localisation il se passe deux phénomènes parallèles qui sont une perte de l'homogénéité du champ des déplacements et le développement des premières microfractures inclinées et localisées dans la bande où va se développer la rupture ;

- de nombreuses méthodes ont été utilisées pour détecter le seuil de localisation dans les roches;

- la sensibilité de ces différentes méthodes semble très variable et il serait bon de lancer une étude visant à les comparer ;

- dans l'état actuel des connaissances, les méthodes les plus adaptées aux laboratoires industriels sont les mesures multiples de déformations ou de déplacements par capteurs ou/et les mesures de vitesse des ondes parce qu'elles emploient des techniques connues bien documentées :

- l'étude précise du seuil de localisation peut parfois amener à remettre en cause les dispositifs d'application des efforts sur les éprouvettes.

Tableau 1 - Résumé des études de localisation. Le type d'essai est indiqué : U pour uniaxial, $T$ pour triaxial de révolution et B pour biaxial.

" $L O C$ " est le rapport de la contrainte axiale au seuil de localisation à la contrainte au pic.

\begin{tabular}{|c|c|c|c|c|}
\hline Authors & Rocks & T. & Meas. Tech. & LOC \\
\hline SCHOLZ (1968) & Granite & $U$. & Emission Acoustique & $92 \%$ \\
\hline FRIEDMAN et al (1970) & $\begin{array}{l}\text { Granite } \\
\text { Calcaire }\end{array}$ & $\begin{array}{l}\text { u. } \\
\text { U. }\end{array}$ & $\begin{array}{l}\text { Microscope } \\
\text { Microscope }\end{array}$ & $\begin{array}{l}99 \% \\
99 \%\end{array}$ \\
\hline THILL (1972) & Marbre & T. & Vitesse ondes & $95 \%$ \\
\hline BRADY (1974) & $\begin{array}{l}\text { Marbre } \\
\text { Granite }\end{array}$ & T. & $\begin{array}{l}\text { Microscope } \\
\text { Microscope }\end{array}$ & $\begin{array}{l}95 \% \\
95 \%\end{array}$ \\
\hline RAO and RAMANA (1974) & $\begin{array}{l}\text { Pyroxénite } \\
\text { Dunite } \\
\text { Serpentine }\end{array}$ & $\begin{array}{l}U . \\
U . \\
U .\end{array}$ & $\begin{array}{l}\text { Vitesse ondes } \\
\text { Vitesse ondes } \\
\text { Vitesse ondes }\end{array}$ & $\begin{array}{l}78 \% \\
75 \% \\
76 \%\end{array}$ \\
\hline HADLEY (1975) & $\begin{array}{l}\text { Granite } \\
\text { Gabbro }\end{array}$ & $\begin{array}{l}\mathrm{T} . \\
\mathrm{T}\end{array}$ & $\begin{array}{l}\text { Mesure déformations } \\
\text { Mesure déformations }\end{array}$ & $\begin{array}{l}90 \% \\
90 \%\end{array}$ \\
\hline LIU and LIVANOS (1976) & Granite & $\mathrm{U}$. & Diffraction fente & $92 \%$ \\
\hline LOCKNER et al (1977) & Granite & T. & Vitesse ondes & $85 \%$ \\
\hline SOBOLEV et al (1978) & Granite & B. & $\begin{array}{l}\text { Vitesse ondes } \\
\text { Holographie } \\
\text { Microscope }\end{array}$ & $80 \%$ \\
\hline SOGA et al (1978) & Granite & T. & $\begin{array}{l}\text { Vitesse ondes } \\
\text { Holographie }\end{array}$ & $60 \%$ \\
\hline KRANZ (1979) & Granite & U. & Microscope & $>87 \%$ \\
\hline WANG (1982) & Granite & T. & Microscope & $99 \%$ \\
\hline YANAGIDANI et al (1985) & Granite & U. & $\begin{array}{l}\text { Emission Acoustique } \\
\text { Mesure déformations }\end{array}$ & $>83 \%$ \\
\hline SANTARELLI (1987) & $\begin{array}{l}\text { Dolomie } \\
\text { Grès }\end{array}$ & T. & $\begin{array}{l}\text { Mesure déformations } \\
\text { Mesure déformations }\end{array}$ & $\begin{array}{l}80-99 \% \\
87-99 \%\end{array}$ \\
\hline
\end{tabular}




\section{UN EXEMPLE D'APPLICATION A UN GRÈS}

\subsection{Technique d'étude et matériel d'essai}

Dans le but de comprendre la technique d'étude choisie, il convient tout d'abord de décrire le matériel d'essai. Il était utilisé lors d'une étude dont le but spécifique n'était pas la détermination du seuil de localisation. Ce matériel consiste en une presse hydraulique servo-contrôlée (Terra Tek System) développant un effort axial maximal de 2,67 MN et une cellule capable de supporter des pressions de $130 \mathrm{MPa}$ et des températures de $400^{\circ} \mathrm{C}$. L'assemblage de l'essai est présenté sur la figure 2 (voir ELLIOTT et BROWN, 1988 pour plus de détails).

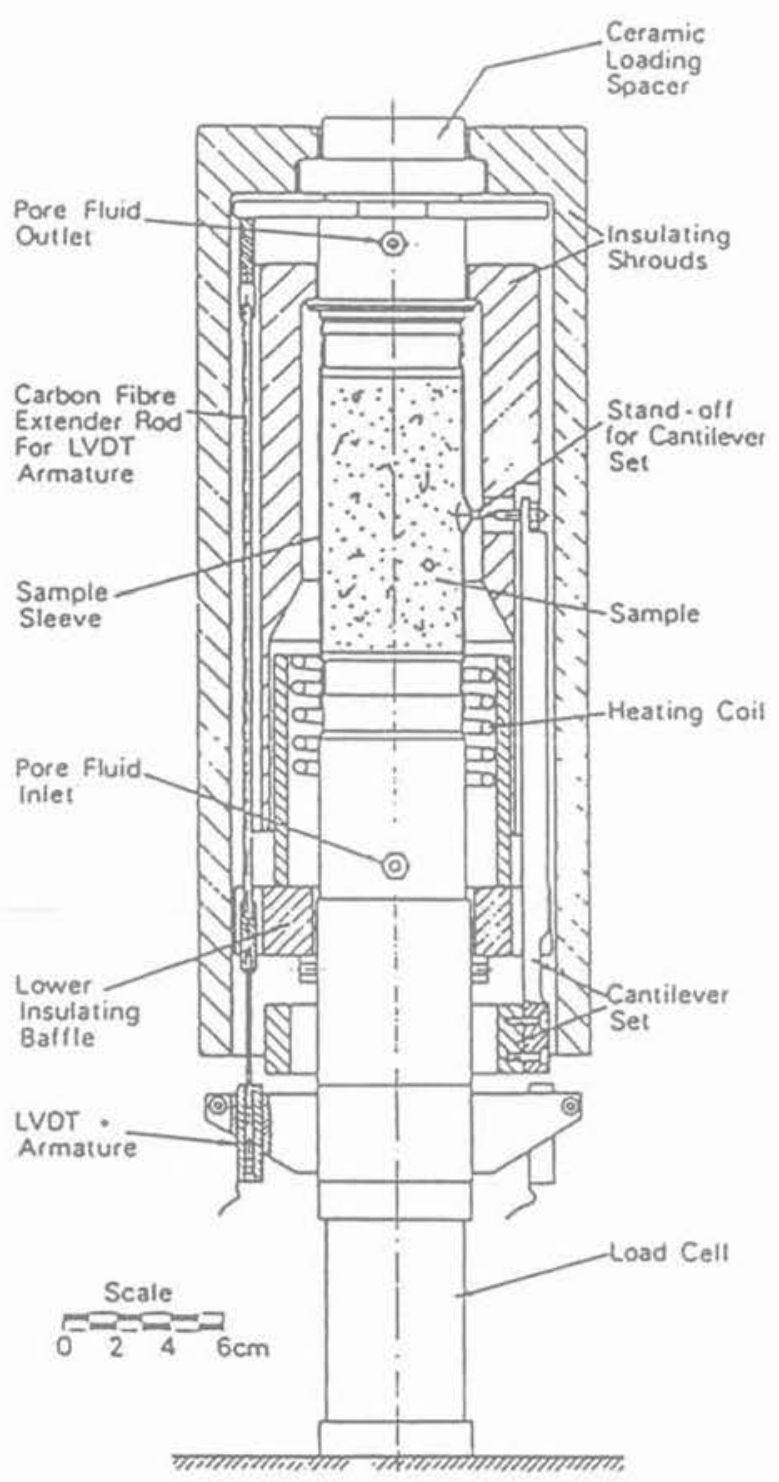

Fig. 2. - Montage de test triaxial

dans la presse "Terra Tek System " d'Imperial College. D'après ELLIOT et BROWN (1988)

Fig. 2. - Stack assembly in the "Terra Tek System » triaxial testing machine at Imperial College.

After Elliot and Brown (1988)
L'instrumentation comporte :

- un capteur de force situé dans la cellule et placé à la base de l'assemblage (load cell) qui mesure le déviateur des contraintes:

- deux LVDT situées de part et d'autre d'un diamètre de l'échantillon qui mesurent le déplacement axial en deux points entre les pièces d'application des efforts ;

- un extensomètre du type cantilever qui, par la mesure de la flexion de poutres, permet d'obtenir le déplacement radial dans le plan horizontal médian de Péchantillon. $\mathrm{Ce}$ dispositif cantilever détermine le déplacement radial dans deux directions à $90^{\circ}$ dans ce plan.

Tous les signaux de mesure sont mis en forme, acquis et traités par un micro-ordinateur. Ces données sont ensuite utilisées pour le tracé automatique de courbes diverses.

Par rapport à l'interprétation classique de l'essai triaxial, il a été tracé la courbe de la déformation radiale $\left(\epsilon_{3,1}\right)$ dans la direction 1 en fonction de la déformation radiale $\left(\epsilon_{3,2}\right)$ dans la direction 2. Pour ce faire, quelques lignes ont été ajoutées au programme graphique du micro-ordinateur.

Sur la base de l'étude de HADLEY (1975), il est possible de conclure que lorsque la courbe $\epsilon_{31}-\epsilon_{32}$ est linéaire, alors la déformation radiale est homogène. On notera que si la pente de la droite est différente de 1 alors la roche n'est pas isotrope. Par contre, lorsque cette courbe n'est pas linéaire, alors la déformation n'est plus homogène dans la mesure où l'échantillon se déforme beaucoup plus dans une direction que dans une autre. Le seuil de localisation de la déformation peut donc être détecté comme correspondant à la limite de linéarité de la courbe $\epsilon_{31}-\epsilon_{32}$. Il est alors facile de remonter aux valeurs des autres paramètres, déviateur des contraintes et déformations axiales, correspondants. La figure 3 représente divers types de profils pour la courbe $\epsilon_{31}-\epsilon_{32}$ tels qu'ils ont été rencontrés sur deux roches (SANTARELLI, 1987).

\subsection{Description de la roche et du programme d'essai}

Le grès de Doddington qui est exploité en carrière à Wooler, Northumberland, Grande-Bretagne appartient au groupe des Fell Sandstones (Carbonifère). La roche est à grains fins et très isotrope. Des analyses chimiques et minérales révèlent que la roche est composée essentiellement de grains de quartz très durs dans une matrice beaucoup plus tendre colorée en rose par des oxydes de fer. Sa porosité est de $23 \%$. Le lot des éprouvettes de grès de Doddington testé est homogène. Les résultats d'une série d'essais uniaxiaux sur 7 échantillons sont présentés sur le tableau 2 .

Les échantillons essayés $(50 \mathrm{~mm}$ de diamètre par $100 \mathrm{~mm}$ de haut) étaient carottés à partir de blocs cubiques de $30 \mathrm{~cm}$ d'arête. Les faces des échantillons étaient rectifiées de façon à les rendre plates ( \pm $0,005 \mathrm{~mm})$ et parallèles $( \pm 0,005 \mathrm{~mm}$ à $5 \mathrm{~cm})$. Les échantillons étaient séchés dans une étuve à $60^{\circ}$ pendant 2 à 3 jours avant d'être équipés pour être essayés. 
Tableau 2 - Résumé des résultats des tests uniaxiaux pour le grès de Doddington. o est la densité sèche, $s_{p}$ la résistance au pic, $E$ le module d'Young et $V$ le coefficient de Poisson.

\begin{tabular}{|l|c|c|c|c|}
\hline & $\rho$ & $\sigma p$ & $E$ & $V$ \\
\hline Valeur moyenne & $2,079 \mathrm{~g} / \mathrm{cm}^{3}$ & $51,4 \mathrm{MPa}$ & $20,73 \mathrm{GPa}$ & 0,39 \\
Déviation standard & $0,0121 \mathrm{~g} / \mathrm{cm}^{3}$ & $0,878 \mathrm{MPa}$ & $0,371 \mathrm{GPa}$ & 0,020 \\
Incertitude & $1,0 \%$ & $3,5 \%$ & $3,6 \%$ & $10,8 \%$ \\
Domaine des mesures & 0,104 & 6,69 & 2,99 & 0,16 \\
Dispersion & $5 \%$ & $13,0 \%$ & $14,4 \%$ & $41 \%$ \\
\hline
\end{tabular}

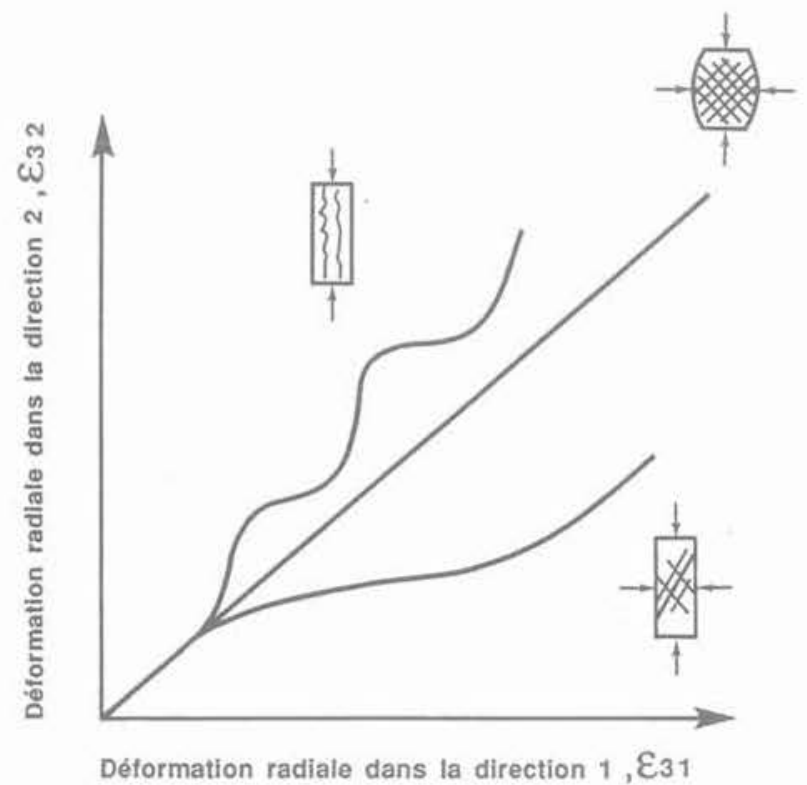

Fig. 3. - Divers types de courbes $\epsilon_{31}-\epsilon_{32}$ rencontrées en essais triaxiaux de révolution.

Fig. 3. - Schematic $\epsilon_{31}-\epsilon_{32}$ curves observed during traxial tests.

Une série de 7 essais uniaxiaux et 21 essais triaxiaux ont été réalisés. Une série de courbes contraintesdéformation typiques sont représentées sur les figures 4 et 5 . Ces essais étaient tous servocontrôlés avec asservissement de la déformation radiale $\left(\epsilon_{31}\right)$ à la vitesse de $654 \times 10^{-6} / \mathrm{s}$, c'est-à-dire environ $0,6 \%$ pour $15 \mathrm{mn}$.

\subsection{Résultats}

Le premier point à envisager est la vérification de la validité du raisonnement présenté au-dessus, à savoir que la limite de linéarité de la courbe $\epsilon_{31}-\epsilon_{32}$ correspond effectivement au seuil de localisation de la déformation dans la roche, c'est-à-dire au seuil d'initiation du processus de rupture. Il est raisonnable de penser que l'ouverture d'une surface de rupture se traduit par une déformation de l'échantillon dans une direction essentiellement orthogonale à la surface de rupture. Si l'on étudie le cas de la rupture selon un plan de cisaillement, l'inclinaison de ce plan sur la

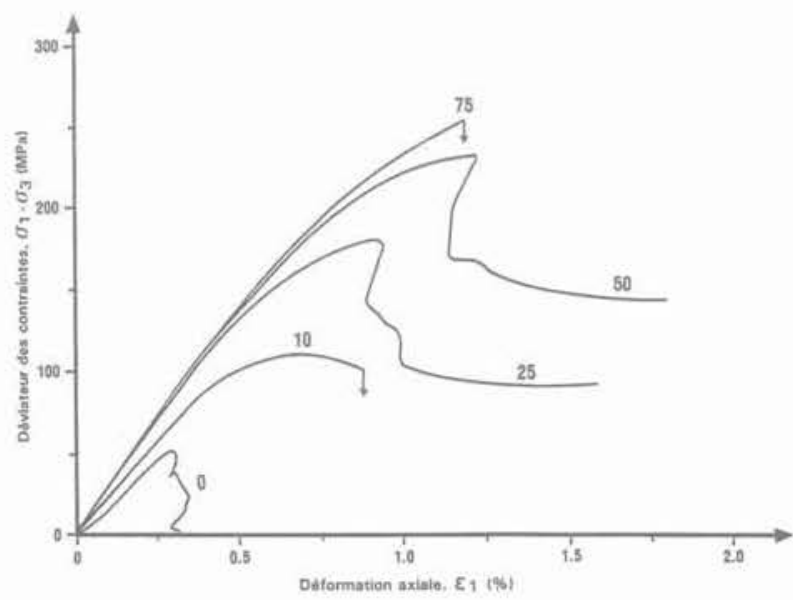

Fig. 4. - Sélection de courbes obtenues lors des essais triaxiaux sur le Grès de Doddington.

Fig. 4. - Selected curves from triaxial tests on Doddington sandstone.

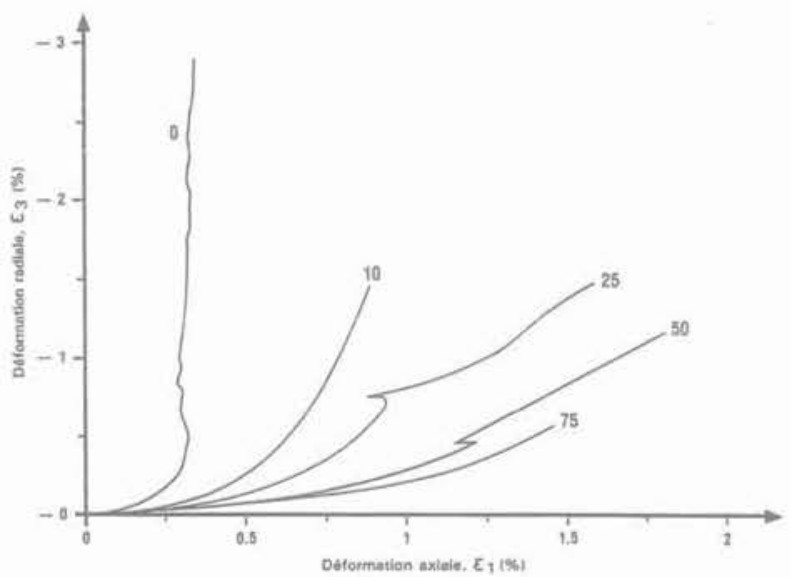

Fig. 5. - Sélection de courbes obtenues lors des essais triaxiaux sur le Grès de Doddington.

Fig. 5. - Selected curves from triaxial tests on Doddington sandstone.

direction de mesure de $\epsilon_{31}$ va déterminer quelle déformation radiale va prédominer. La courbe $\epsilon_{31}-$ $\epsilon_{32}$ devrait donc présenter une allure semblable à celle de la courbe correspondante de la figure 3. 
C'était le cas pour les 21 essais triaxiaux sur le grès de Doddington. Par contre, dans le cas des essais uniaxiaux où l'échantillon développe une série de fractures verticales (rupture en colonnettes), la courbe $\epsilon_{31}$ - $\epsilon_{32}$ devrait avoir un aspect beaucoup plus irrégulier avec tantôt $\epsilon_{31}$ tantôt $\epsilon_{32}$ s'accroissant en priorité suivant que la rupture se développe dans une direction orthogonale à la direction 1 ou 2. Les 7 essais uniaxiaux sur le grès de Doddington donnaient des courbes $\epsilon_{31}-\epsilon_{32}$ ayant cette allure. Des résultats semblables ont été obtenus par SANTARELLI (1987) dans le cas d'une dolomie.

On doit retenir de ces observations qu'il est possible de détecter le seuil de localisation pendant un essai uniaxial ou triaxial de révolution en utilisant un matériel de mesure simple.

On peut maintenant reporter le point ainsi repéré, sur les courbes déviateurs des contraintes-déformation axiale et déformation radiale-déformation axiale comme ceci est illustré par la figure 6 .

On observe alors que:

- le seuil de localisation est situé légèrement avant le pic ;

- la plus grande partie de la dilatance que subit l'échantillon se situe après le seuil de localisation. Cette observation expérimentale semble importante au niveau théorique si l'on se reporte à l'étude de VARDOULAKIS, SULEM et GUENOT (1988) ;

- les courbes $(\sigma 1-\sigma 3)$ - $\epsilon 1$ et $\epsilon 3-\epsilon 1$ présentent parfois des points anguleux au seuil de localisation (fig. 6). Ce point de discontinuité des tangentes aux courbes semble donc correspondre à une discontinuité de régime des contraintes et déformations. On notera que ceci est expliqué par la théorie des bifurcations ; - enfin, on notera que la partie des courbes sur laquelle on peut étalonner une loi de comportement, est très limitée. Elles correspondent à un matériau peu dilatant et écrouissant.

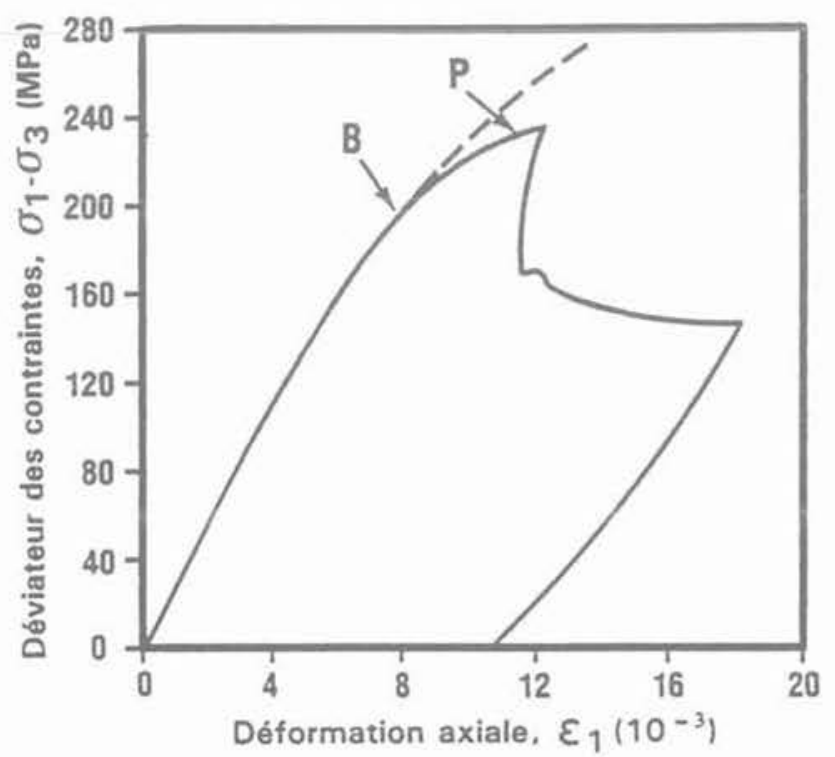

La figure 7 représente l'évolution du rapport des contraintes axiales au seuil de localisation et au pic, en fonction de la pression de confinement. Elle montre que sur le domaine de pression de confinement étudié $(0$ à $90 \mathrm{MPa})$, ce rapport diminue légèrement. Cela signifie que la localisation a tendance à se produire de plus en plus tôt avant le pic lorsque l'on augmente la pression de confinement. La figure 7 présente un véritable critère de localisation au même titre où il $\mathrm{y}$ avait un critère de pic ou un critère de plasticité. SANTARELLI et BROWN (1988) proposent l'équation :

$$
\sigma_{\mathrm{loc}} / \sigma_{\mathrm{p}}=0,98-7 \times 10^{-4} \sigma_{3}
$$

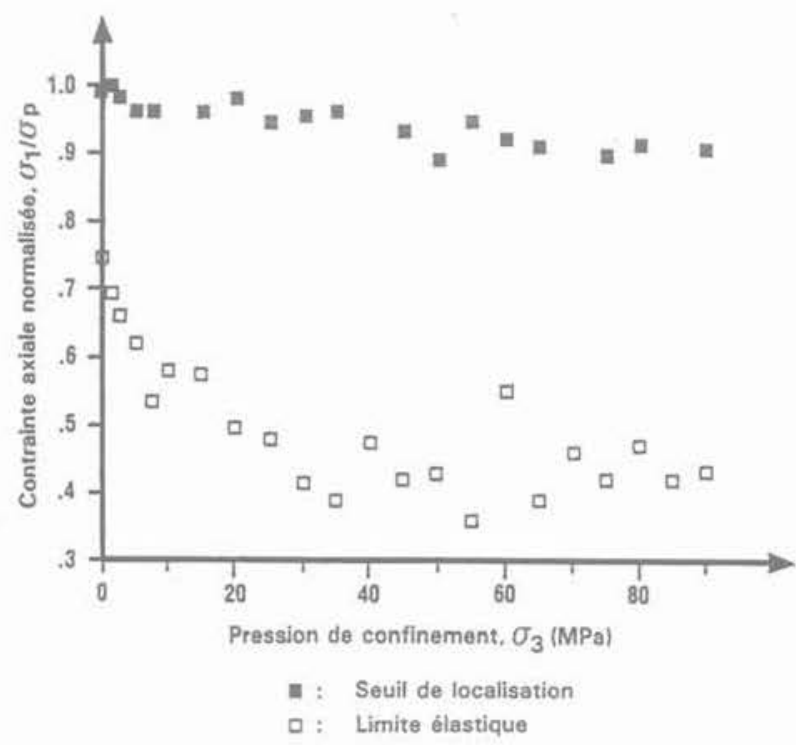

Fig. 7. - Evolution du rapport " $L O C$ " avec la pression de confinement.

Fig. 7. - Evolution of the LOC ratio with confining pressure.

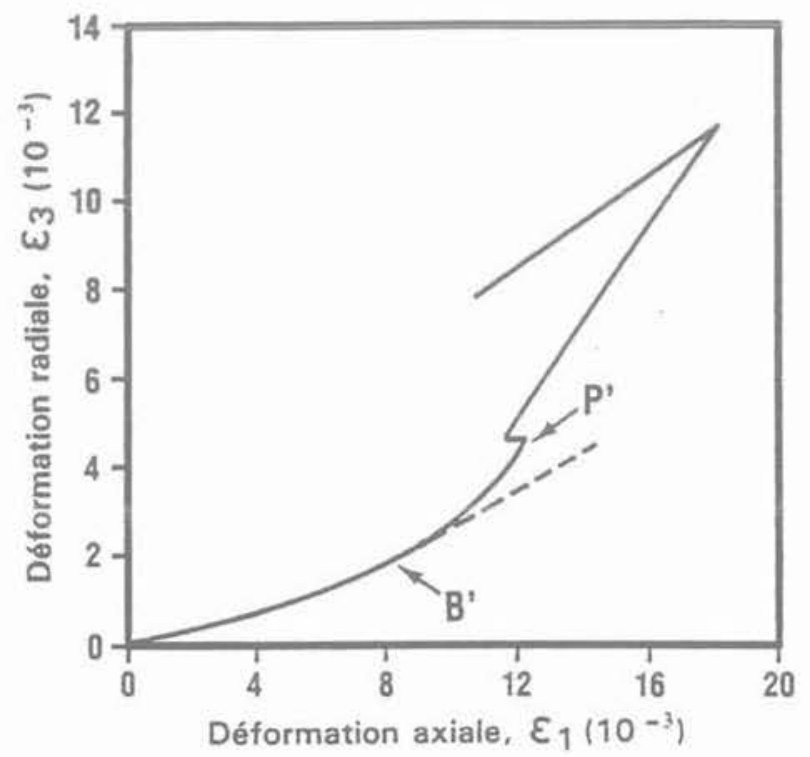

Fig. 6. - Le pic et la bifurcation dans un grès essayé en conditions triaxiales avec 50 MPa de pression de confinement. Fig. 6. - Pic and bifurcation threshold measured on a specimen tested under $50 \mathrm{MPa}$ confining pressure in triaxial conditions. 
pour décrire le nuage des points de la figure 7 où $\sigma_{\text {loc }}$ est la contrainte axiale au seuil de la localisation avec la résistance au pic op qui peut être décrite par :

$$
\sigma_{\mathrm{p}}=51,1\left[1+4,37\left(\sigma_{3} / 51,4\right)^{0,72}\right]
$$

où $\sigma_{\mathrm{p}}$ et $\sigma_{3}$ sont exprimés en $\mathrm{MPa}$.

Enfin, la figure 8 présente les points déviateurs des contraintes en fonction du déviateur des déformations au seuil de localisation pour les différentes pressions de confinement. La linéarité de cette courbe peut être interprétée en termes élasto-plastiques. Le module élastique de cisaillement correspondrait alors à la pente de cette droite. L'intersection de la droite avec l'axe horizontal est une déformation plastique. Il serait alors possible de conclure que le seuil de localisation est atteint pour un déviateur des déformations plastiques constant.

\subsection{Synthèse de l'exemple d'application}

On retiendra les points suivants de l'étude présentée ci-dessus :

- la détermination du seuil de localisation pendant l'essai triaxial de révolution peut être faite simplement par l'utilisation de plusieurs capteurs de déplacement ou de déformation ;

- elle consiste à tracer les déformations radiales les unes en fonction des autres. La limite de linéarité de ces courbes correspond au seuil de localisation;

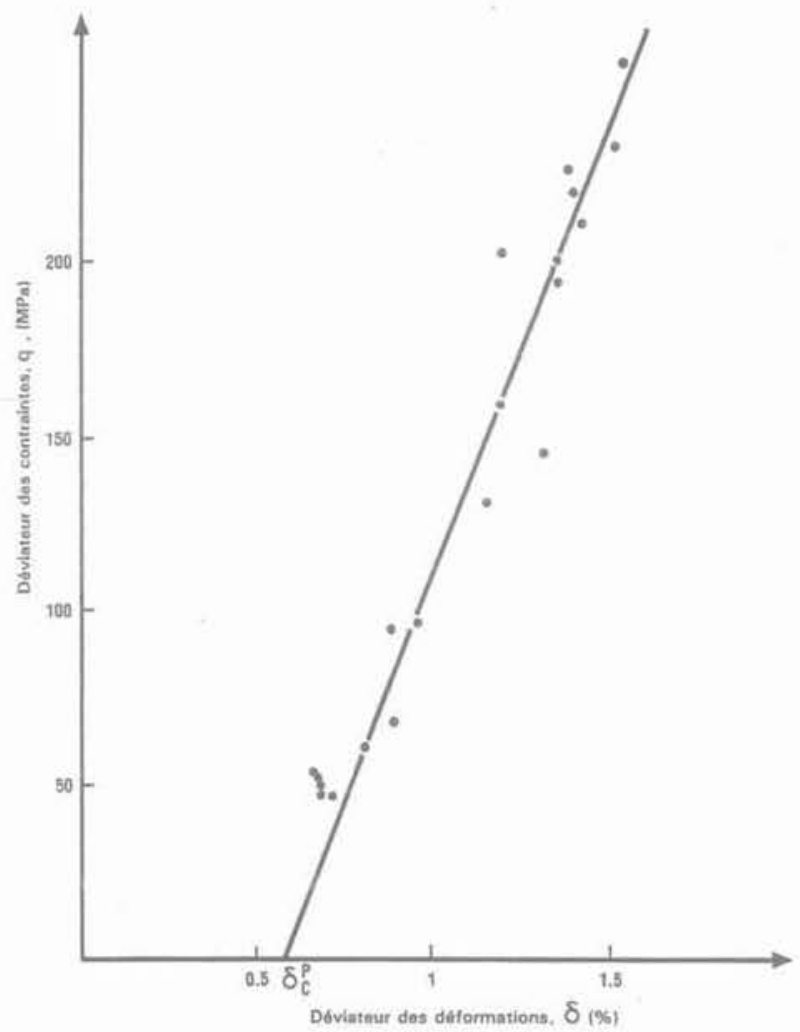

Fig. 8. - Déviateur des contraintes en fonction du déviateur des déformations au seuil de localisation pour différentes valeurs de la pression de confinement.

Fig. 8. - Deviatoric stress as deviatoric strain at the threshold of bifurcation for various confining pressures.
- le seuil de localisation correspond parfois à un point anguleux des courbes efforts-déformations, c'està-dire à un changement de régime des contraintes et déformations ;

- le comportement pré-localisation des échantillons testés est caractérisé par une faible dilatance et un écrouissage ;

- le seuil de localisation est situé légèrement avant le pic et le rapport des contraintes au seuil de localisation et au pic décroît légèrement lorsque la pression de confinement s'accroît ;

- la courbe déviateur des contraintes-déviateurs des déformations au seuil de localisation pour les divers essais est très régulière et suggère que dans une certaine interprétation, la localisation se produirait lorsque le déviateur des déformations plastiques dépasse un niveau critique indépendant de la pression de confinement.

\section{CONCLUSION}

Les résultats présentés ci-dessus indiquent que la détermination expérimentale du seuil de localisation lors de l'essai triaxial de révolution est aisée. Au niveau du laboratoire industriel, une telle détermination est donc possible. Elle revêt tout son intérêt dans la mesure où elle détermine la partie des courbes contraintes-déformations qui est valide et qui de ce fait peut être utilisée pour étalonner les lois de comportement. Les résultats présentés ci-dessus montrent tous que le seuil de localisation se situe avant le pic; c'est-à-dire que les roches concernées sont faiblement dilatantes et écrouissables.

Néanmoins, une synthèse des techniques disponibles pour étudier le seuil de localisation a montré que les nombreuses techniques existantes ne sont pas équivalentes. En effet, elles ont, non seulement, des champs d'application différents mais aussi des sensibilités différentes. Cela signifie que deux méthodes utilisées lors d'un même essai peuvent donner des valeurs du seuil de localisation différentes. Pourtant dans la mesure où la presse d'essais peut, elle aussi, avoir une forte influence sur le seuil de localisation, il serait intéressant de lancer une campagne systématique de comparaison et d'évaluation de ces techniques.

Enfin, la méthode de mesure illustrée dans cet article (exploitation des mesures de déformations) a été utilisée avec succès pour déterminer le point de bifurcation entre le mode de déformation homogène et le mode de rupture en colonnette d'une part, et le mode de déformation homogène et le mode de rupture par plan de cisaillement d'autre part. Il a été montré (SANTARELLI et BROWN, 1988) que cette technique ne pouvait être employée pour la détection de la mise en tonneau de l'échantillon ; c'est-à-dire le cas où la courbe déviateur des contraintes-déformation axiale ne présente pas de pic. Dans ce cas, une technique semblable peut être utilisée qui consiste à com- 
parer la déformation volumique globale mesurée directement, à la déformation volumique, calculée à partir des déformations axiales et radiales en faisant l'hypothèse de la déformation du cylindre parfait. Cette dernière méthode a été utilisée avec succès par ELLIOT (1982) avec un calcaire. Une telle technique peut s'avérer très importante pour toute une classe de problèmes industriels où l'on a besoin d'un * critère de rupture » qui ne peut pas être obtenu par des essais de laboratoire.

\section{REMERCIEMENTS}

L'auteur remercie la Société Nationale Elf Aquitaine Production, qui a financé cette recherche, d'avoir autorisé la présente publication. Il remercie aussi le Professeur E.T. BROWN pour ses suggestions multiples et les Docteurs V. MAURY, A. GUENOT et D. FOURMAINTRAUX pour leurs commentaires sur le présent article.

\section{BIBLIOGRAPHIE}

BRADY B.T. (1974), Theory of Earthquakes. I. A scale independant theory of rock failure. Pure Appl. Geophys., 112, pp. 701-725.

DESRUES J. (1987), Naissance des bandes de cisaillement dans les milieux granulaires: expérience et théories. Manuel de Rhéologie des Géomatériaux, (F. DARVE, ed). Presses de l'Ecole Nationale des Ponts et Chaussées, pp. 279-298.

ELLIOT G.M. (1982), An investigation of a yield criterion for porous rock. $\mathrm{PhD}$ thesis, Imperial College, $363 \mathrm{pp}$.

ELLIOT G.M., BROWN E.T. (1988), Laboratory measurements of the thermo hydro-mechanical properties of rock. Q.J. Engng. Geol., 21, (à paraitre).

FOURMAINTRAUX D. (1970), Contribution de la pétrographie à létude physique et mécanique des roches. Thèse de Doctorat, $134 \mathrm{pp}$.

FRIEDMAN M. PERKINS R.D., GREEN S.J. (1970), Observation of brittle deformation features at the maximum stress of Westerly Granite and Solenhofen Limestone. Int. J. Rock Mech. Min. Sci., 7, pp. 297-306.

GUENOT A., SANTARELLI F.J. (1988), Borehole stability : a new challenge for an old problem. In key questions in Rock Mechanics, (P.A. CUNDAL, R.L. STERLING \& A.M. STARFIELD, eds), Proc. $29^{\text {th }}$ Symp. Rock Mech., A.A. Balkema, Rotterdam, pp. 453-460.

HADLEY K. (1975), Azimuthal variation of dilatancy. J. Geophys. Res., 80, pp. $4845-4850$.

KULHAWY F.M., Stress deformation properties of rock and rock discontinuities. Engng Geol., 9, pp. 327-350.

LIU H.P., LIVANOS A.C.R. (1976), Dilatancy and precursary bulging along incipient fracture zones in uniaxially compressed Westerly granite. J. Geophys. Res., 81, pp. 3495-3510.
LOCKER D.A., WALSH J.B., BYERLER J.D. (1977), Changes in seismic velocity and attenuation during deformation of granite. J. Geophys. Res., 82, pp. 5374-5478.

MAURY V. (1987), Observations, recherches et résultats récents sur les mécanismes de rupture autour de galeries isolées. Rapport de la Commission SIMR sur les mécanismes de rupture autour d'ouvrages souterrains. Proc. $6^{\text {th }}$ ISRM Congr. (G. HERGET et S. VONGPAISAL, eds), BALKEMA, 2, pp. 1119-1128.

PATERSON M.S. (1978), Experimental rock deformation. The Brittle Field. Springer Verlag, $254 \mathrm{pp}$.

RAMEZ M.R.H. (1967), Fractures and the strength of a sandstone under triaxial compression. Int. J. Rock. Mech. Min. Sci., 4, pp. 257-268.

RAO M.V.M.S., RAMANA V.M. (1974), Dilatant behaviour of ultra-mafic rocks during fracture. Int. J. Rock Mech. Min. Sci. \& Geomech. Abstr., 11, pp. 193-203.

READ H.E., HEGEMEIER G.A. (1984). Strain softening of rock, soil and concrete - a review article. Mech. Mat., 3, pp. 271-294.

SANTARELLI F.J. (1987), Theoretical and experimental investigation of the stability of the axisym. metric wellbore. $\mathrm{PhD}$ thesis, Imperial College, $472 \mathrm{pp}$.

SANTARELLI F.J., BROWN E.T. (1988), Failure of three sedimentary rocks in triaxial and hollow cylinder compression tests. (Soumis à l'Int. J. Rock Mech. Min. Sci. \& Geomech. Abstr.).

SCHOLZ C.H. (1968), Experimental study of the fracturing process in brittle rock. J. Geophys. Res., 73, pp. 1447-1454.

SOBOLEV G., SPETZLER H., SALOV B. (1978), Precursors to failure in rocks while undergoing anelastic deformations. J. Geophys. Res., 83, pp. 1775-1784.

SOGA N., MIZUTANI H., SPETZLER H., MARTIN III R.J. (1978), The effect of dilatancy on veloctity anisotropy in Westerly granite. J. Geophys. Res., 83, pp. 4451-4458.

SONDERGELD C.M., ESTEY L.H. (1981), Acoustic emission study of microfracturing during the cyclic loading of Westerly Granite. J. Geophys. Res., 86, pp. 2915-2924.

SPETZLER H., MARTIN III R.J. (19747, Correlation of strain and velocity during dilatancy. Nat., 252, pp. 30-31.

SPETZLER H., SCHOLZ C.H., LU C.J. (1974), Strain and creep measurements on rocks by holographic interferometry. Pure Appl. Geophys., 112, pp. $571-581$.

THILL R.E. (1972), Acoustic methods for monitoring failure in rock. New Horizons in Rock Mechanics, Proc. $14^{\text {th }}$ U.S. Symp. Rock Mech., (H.R. HARDY et R. STEPHANKO, eds.), Am. Soc. Civ. Engrs., pp. 649-687.

TORRENTI J.M. (1987), Communication à la réunion du GRECO Géomatériaux (Décembre). 
TOURENQ (1967), Mise en évidence de la microfissuration. C.R. Soc. Géol. France, fasc. 5.

VARDOULAKIS I.G. (1984), Rock bursting as a surface instability phenomenon. Int. J. Rock Mech. Min. Sci. \& Geomech. Abstr., 21, pp. 137-144.

VARDOULAKIS I.G., SULEM J., GUENOT A. (1988) Stability of deep borehole as a bifurcation phenomenon. Int. J. Rock Mech. Min. Sci. \&
Geomech. Abstr. (à parattre).

WONG T.F. (1982), Micromechanics of faulting in Westerly granite. Int. J. Rock Mech. Min. Sci. Geomech. Abstr., 19, pp. 49-64.

YANAGIDANI T., EHARA S., NISHIZAWA O., KUSUNASE K. (1985), Localization of dilatancy in Ohshima Granite under constant uniaxial stress.

J. Geophy. Res., 90, pp. 6840-6858. 